\title{
Kawasaki disease: echocardiographic features in 91 cases presenting in the United Kingdom
}

\author{
A Suzuki, E J Tizard, V Gooch, M J Dillon, S G Haworth
}

\begin{abstract}
Ninety one patients with Kawasaki disease were examined by cross sectional echocardiography between 1980 and 1988. In the 75 patients evaluated during the acute phase of the illness (the first month), the first echocardiographic examination was carried out at a mean time of 16 days (range 5-30) and coronary arterial lesions were seen in 21 (28\%). Two patients with medium sized aneurysms had myocardial infarctions, and one died. Coronary arterial lesions persisted in $17(23 \%)$ patients, most often in younger children. The remaining 16 patients were examined from one month to four years after their acute illnesses, and this group did not have coronary arterial abnormalities. Seven patients with coronary artery lesions have reached school age and require regular echocardiographic examination and exercise electrocardiography. Selective coronary arteriography may be indicated in some patients to identify coronary artery stenosis, which the Japanese experience has shown may progress for several years after the acute illness.
\end{abstract}

It has recently become obvious that Kawasaki disease is not always self limiting or benign, as previously reported. ${ }^{1}$ Patients without coronary artery abnormalities on echocardiography should be followed up for a long period as well as those known to have cardiac lesions. Even if patients did not have dilated coronary arterial lesions when they were acutely ill, or if they had aneurysms that had disappeared on subsequent echocardiographic examination, there is still a possibility that the disease may progress. ${ }^{2}$ Coronary arterial intimal proliferation that developed during the acute illness could progress and cause stenotic lesions, ${ }^{34}$ or juvenile arteriosclerosis. $^{2}$ These conclusions are based on work done in Japan.

The present study was carried out to establish the incidence and severity of coronary arterial abnormalities in children living in the United Kingdom, with the intention of establishing guidelines about the most appropriate method of following up patients with Kawasaki disease. We therefore reviewed the cross sectional echocardiographic findings of all patients with Kawasaki disease examined by a paediatric cardiologist between 1978 and 1988 at this hospital. The non-cardiological features of most of these patients are described in a companion paper. ${ }^{5}$

\section{Patients and methods}

Between 1978 and 1988 cross sectional echocardiographic examinations were carried out on 91 of 93 children in whom a diagnosis of Kawasaki disease or suspected Kawasaki disease had been made at the onset of their illness (fig 1). All became ill in the United Kingdom. All six of the diagnostic criteria of Kawasaki disease were fullfilled in 49 patients, and five criteria were met in 29 cases. ${ }^{6}$ There were nine cases in which only four criteria were fulfilled, but four of these developed coronary arterial lesions that were seen on echocardiography during the acute illness, and in five the clinical picture was typical of the disease. The remaining six patients were referred for echocardiographic assessment after the acute illness but were said to have had clinical courses typical of the disease. There were 49 boys and 44 girls (sex ratio $1 \cdot 1: 1$ ). The age of onset of the illness ranged from 3 months to 11 years. During the acute phase most of the patients $(n=80,86 \%)$ were treated with aspirin, and the remainder were given dipyridamole $(\mathrm{n}=33,35 \%)$, gammaglobulin $(\mathrm{n}=16,17 \%)$, steroids $(n=5,5 \%)$, or prostacyclin. Some were given antibiotics alone.

In this study the acute phase of the disease was defined as being within one month of the onset of the illness. A cardiac sequela was defined as a coronary arterial lesion which remained for more than one month after the acute onset.

The echocardiographic findings were reviewed in 91 cases; the remaining two patients had presented in 1977 and 1978 when it was not possible to assess coronary arterial anatomy by

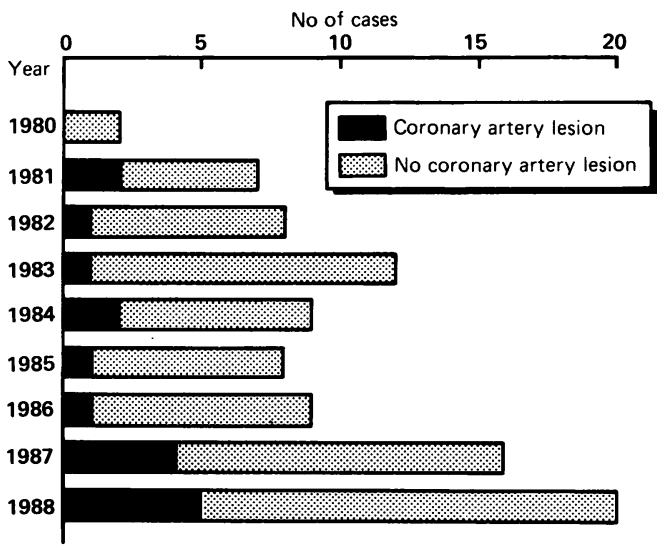

Figure 1 Number of patients examined by cross sectional echocardiography between 1980 and 1988, with and without abnormalities of the coronary arteries. 
cross sectional echocardiography. Seventy five patients have been studied repeatedly since the acute phase of the illness.

The dilated lesions in the coronary arteries were classified according to the standardised diagnostic criteria of the research committee on Kawasaki disease of the Japanese Ministry of Health and Welfare. ${ }^{6}$ A 'large' aneurysm was defined as an aneurysm the diameter of which was four times or more that of the adjacent intact coronary artery. The diameter of a 'medium' aneurysm was between one and a half and four times, and a 'small' aneurysm was less than one and a half times that of the intact coronary artery. Aneurysms change in size during the course of the disease and the measurement given is the largest diameter that was measured.

In all the examinations either a $5 \mathrm{MHz}$ short focus mechanical sector scanner or a $7 \cdot 5 \mathrm{MHz}$ mechanical sector scanner was used.

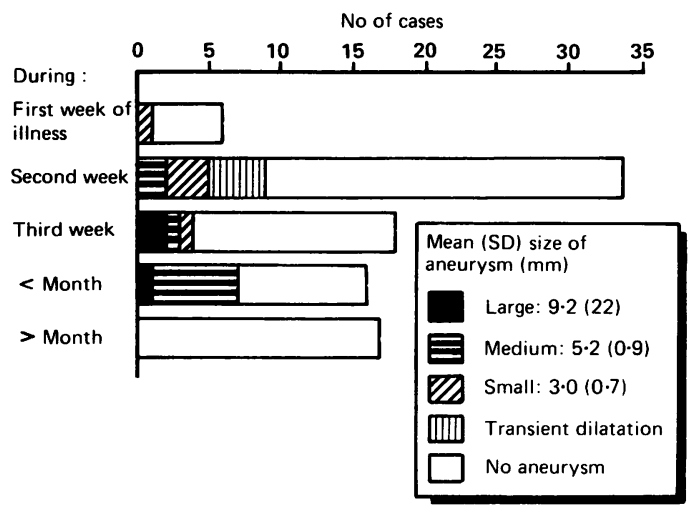

Figure 2 Time during the acute illness at which the first echocardiographic study was carried out, with the principal findings. Three, nine, and nine patients had large, medium, and small aneurysms, respectively.

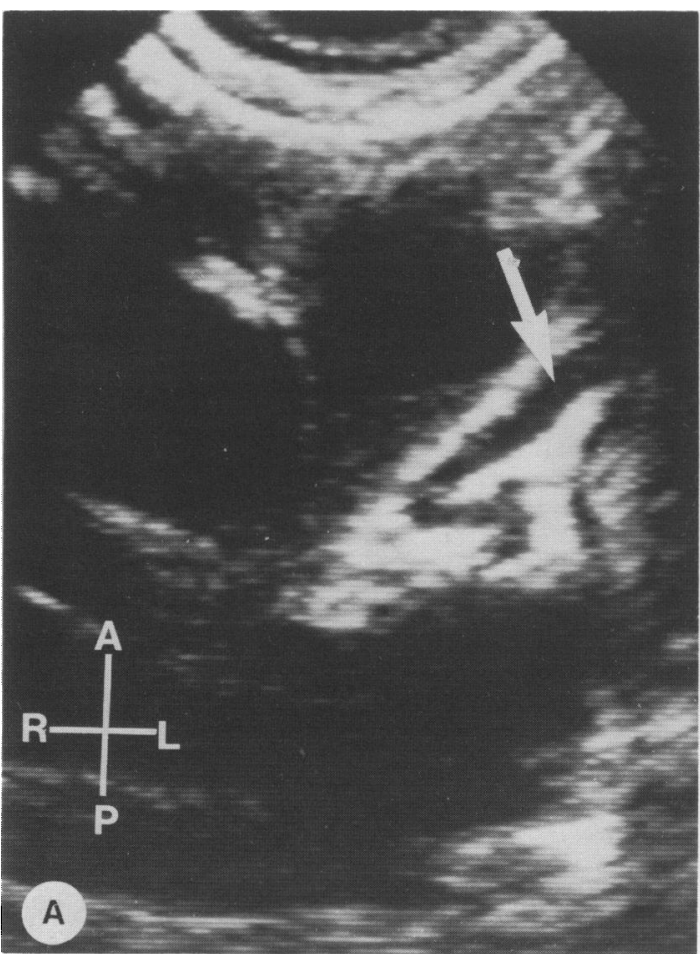

Figure 3 Patient aged 10 years. (A) Parasternal short axis view showing diffuse dilatation of left anterior descending coronary artery and circumflex branch, showing early aneurysmal formation (arrow). The aneurysm proved to be transient. (B) Normal study: parasternal short axis view showing left coronary artery. $(R$, right; $L$, left; $A$, anterior; $P$, posterior.)

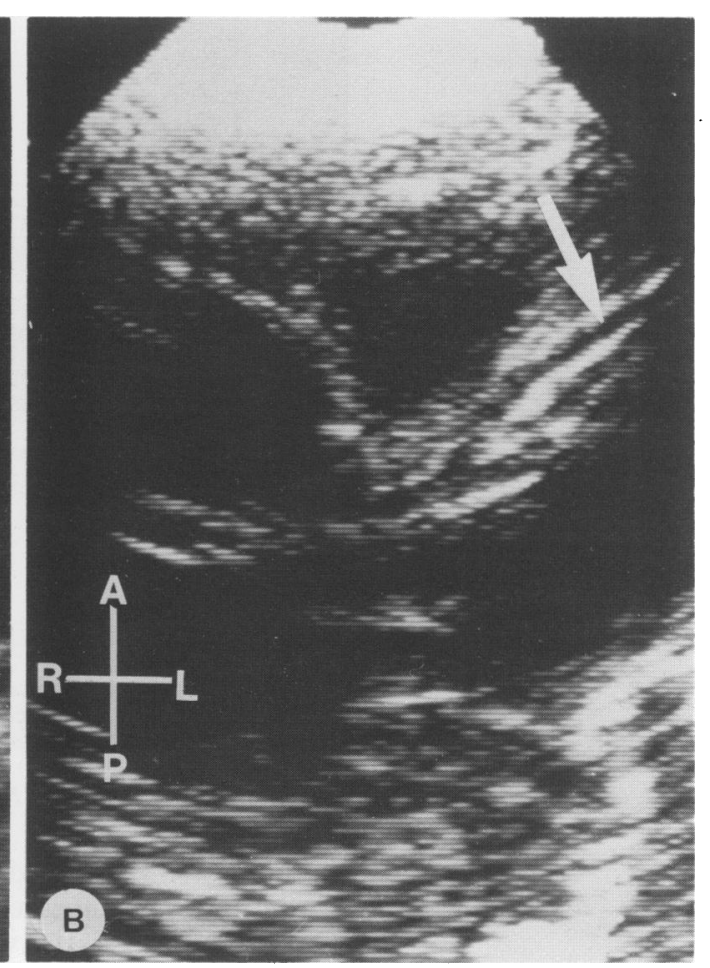

FINDINGS ON CROSS SECTIONAL

ECHOCARDIOGRAPHY

Seventy five children were examined by cross sectional echocardiography during the acute phase of the disease (fig 2). Sixteen children were first examined more than one month after the onset of the disease (range 32 days -4 years). Coronary artery lesions were not seen in the group of patients who presented late (fig 2).

Of the 75 patients first examined during the acute phase, cross sectional echocardiography was carried out 5-30 days after the onset of symptoms (mean (SD) $16.0(6.5)$ ) (fig 2). The first echocardiographic examination was made dren, and one of these already had a small aneurysm of the left coronary artery. Thirty five children were first examined during the second week, and nine of these had coronary artery lesions. Slight dilatation was seen in four of these nine patients, and was transient (fig 3). It disappeared within the acute phase and affected only one vessel in three children, and two vessels in one child. Of the remaining five patients, small aneurysms in three children and medium sized aneurysms in two persisted for more than a month after the onset of the illness. During the third week of the disease 18 cases were examined for the first time and coronary aneurysms were detected in four children. The aneurysms were small in one, of medium size in one, and large in two (fig 4). A massive pericardial effusion was seen in one child.

The first examination was performed between three and four weeks after the onset of the illness in 16 children. Seven of the 16 cases (44\%) had aneurysms on the first examination; they were of medium size in six children and large in one. Thus the incidence of coronary during the first week of the illness in six chil- 

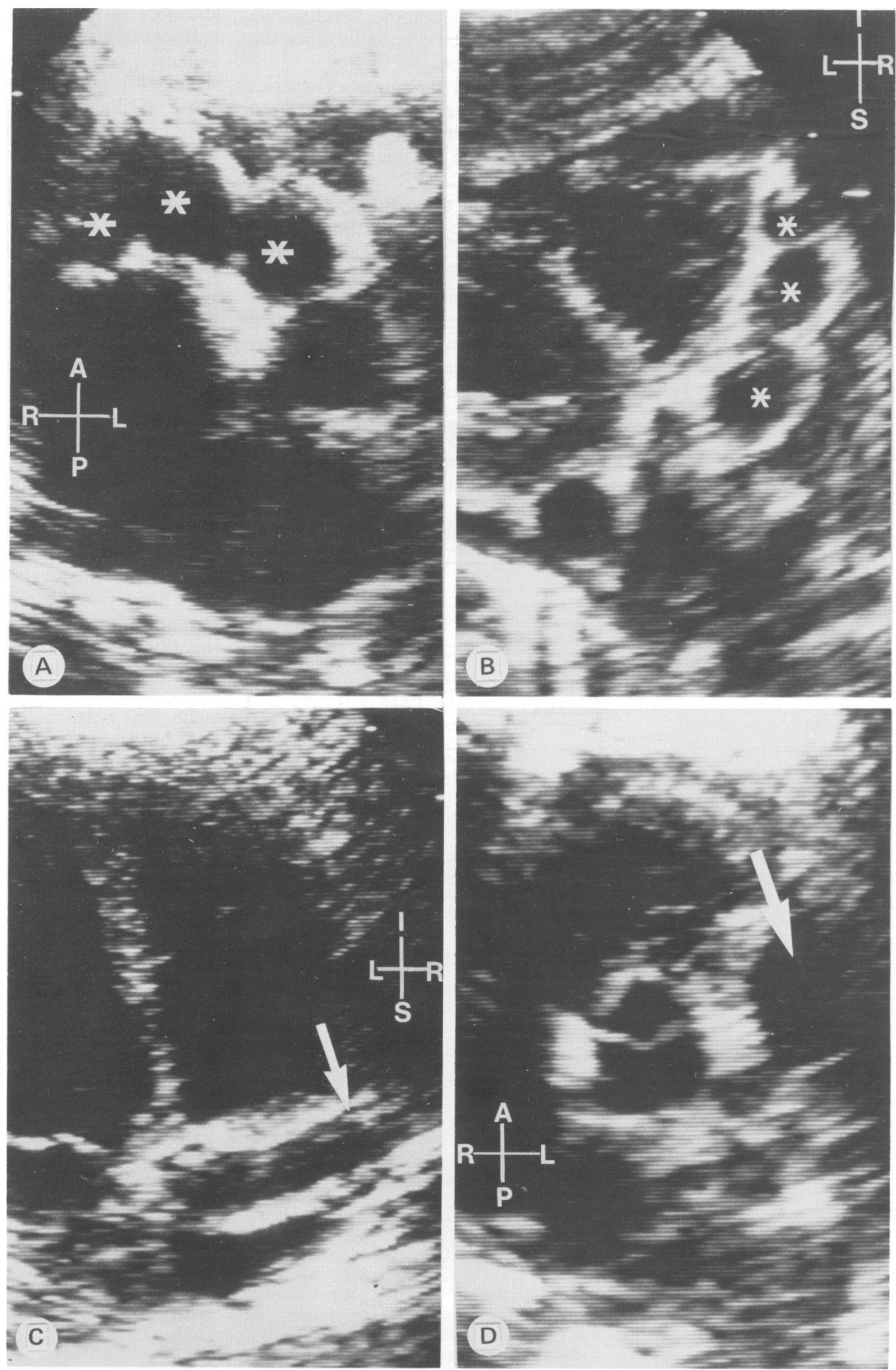

Figure 4 Patient aged $1 \cdot 3$ years. (A) Parastemal short axis view showing three large aneurysms in proximal right coronary artery $\left({ }^{*}\right)$. These aneurysms were still present eight months after presentation. (B) Subcostal view of aneurysms shown in $(A)$. Note that the anatomic orientation is different from that in $(A)$. (C) Apical four chamber view of right coronary artery showing generalised dilatation (arrow). See fig $3(B)$ for control. (D) Parasternal short axis view showing large aneurysm at origin of left coronary artery (arrow). ( $R$, right; $L$, left; $A$, anterior; $P$, posterior; $I$, inferior; $S$, superior.)

arterial lesions increased during the first month of the illness, being greatest during the fourth week. Three vessels were affected in nine patients, two in seven, and one in five.

In one 3 month old child cross sectional echocardiography showed medium sized aneurysms
$(5.0 \mathrm{~mm})$ that extended into the peripheral branches of both coronary arteries and there was ventricular hypokinesis extending from the interventricular septum to the apex of the left ventricle that was associated with marked thinning of the apical myocardium (fig 5), indicat- 

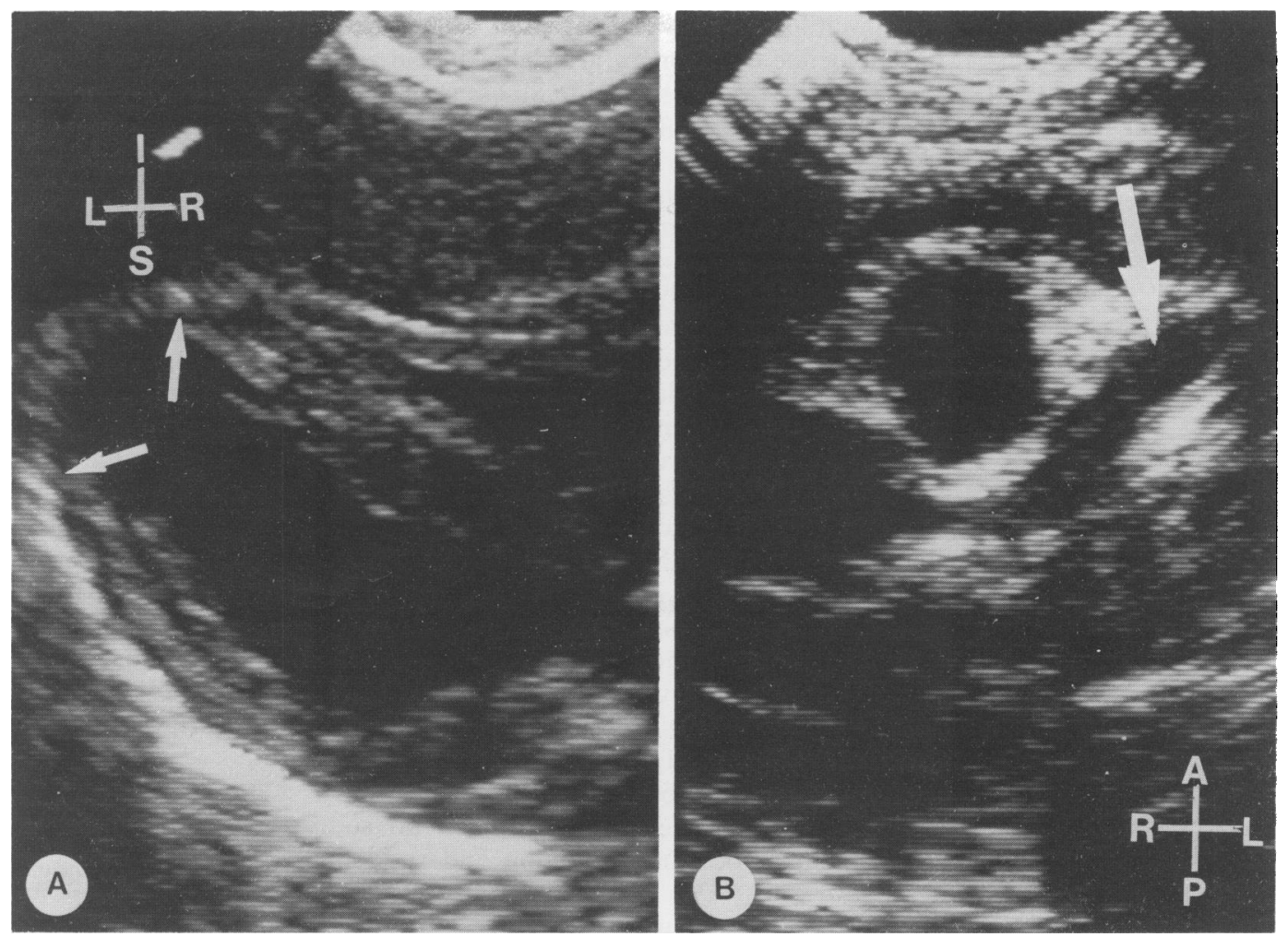

Figure 5 Patient aged 3 months. (A) Subcostal, five chamber view to show thin walled apex of left ventricle (between arrows). (B) Parasternal short axis view showing dilated left coronary artery (arrow), which has irregular margins. ( $R$, right; $L$, left; $A$, anterior; $P$, posterior; $I$, inferior; $S$, superior.)

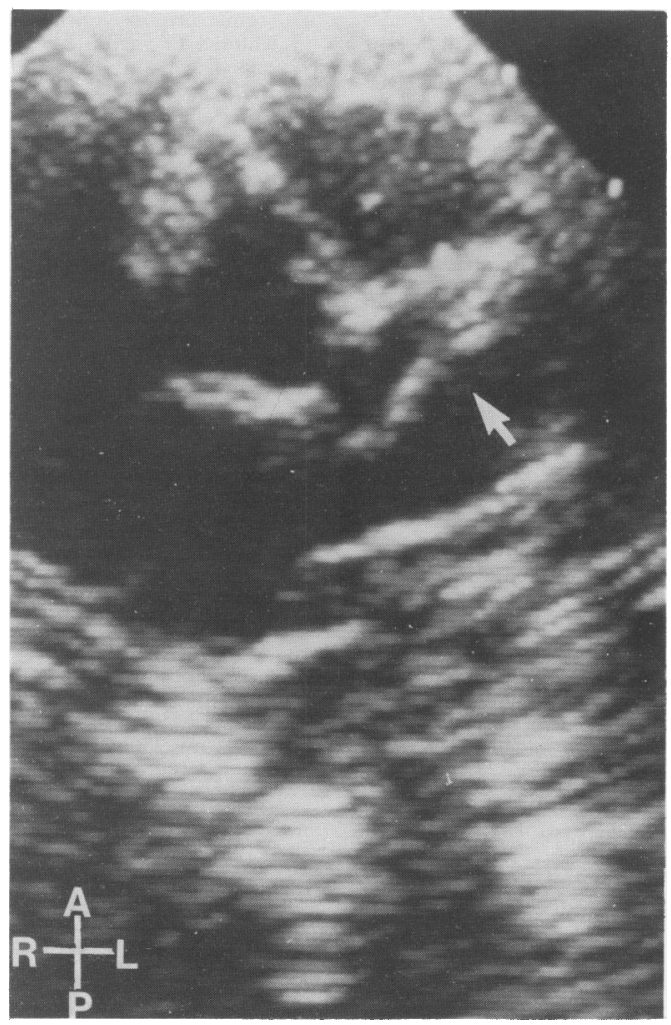

Figure 6 Patient aged 7 months. Medium sized aneurysm at bifurcation of left coronary artery (arrow), on the 24th day of the illness. She died three days later of occlusion of the left anterior descending coronary artery. $(R$, right; $L$, left;

$A$, anterior; $P$, posterior.)

ing obstruction of the left anterior descending coronary artery. A patient aged 7 months had a medium sized aneurysm $(3.4 \mathrm{~mm})$ at the bifurcation of the left coronary artery (fig 6). She had a myocardial infarction on the 27 th day of the illness from which she died, and at necropsy the left anterior descending coronary artery was found to be occluded.

Twenty one children developed coronary artery lesions during the acute phase $(28 \%)$. Cardiac sequelae were seen in 17 cases, 11 of the 44 girls $(25 \%)$ and six of the 49 boys (12\%). A third of the children less than 1 year old developed coronary arterial sequelae, compared with four (27\%) in those between the ages of 1 and 2 years, two (7\%) in those between 2 and 3, and two $(8 \%)$ in the older children.

Seven children in whom a coronary artery lesion was seen on echocardiographic examination during the acute phase have now reached school age. Of the 93 patients, 37 had repeat echocardiograms between six months and seven years after the acute illness. Eight children still had aneurysms 0.5 to 2.25 years later, and all were symptom free. These eight patients had had acute illnesses at a mean age of 0.8 years (range 0.3-2 years) and were re-examined at a mean age of $2 \cdot 2$ years (range $0 \cdot 8-4 \cdot 2$ years). We are not aware of any late deaths.

\section{Discussion}

The number of patients with Kawasaki disease referred to this hospital for echocardiographic examination has increased gradually during the past 10 years without there being a conspicuous outbreak of the disease. ${ }^{5}$ The referral rate increased as soon as it became apparent that coronary arterial aneurysms can be seen on cross sectional echocardiography.

Kawasaki disease was first reported in Japan in $1967,{ }^{1}$ and the first report of a case occurring in the United Kingdom was in $1970 .^{7}$ Comparing the cardiological pictures in Japanese and 
British children, ${ }^{\gamma}$ the present study shows that the incidence of coronary arterial sequelae and the age at the time of the acute illness is similar. Rather more girls than boys were affected in the study group, however, than in Japan. Echocardiography was first used for the detection of coronary artery lesions in $1977 .{ }^{9}$ Two patients in the present study presented shortly after this and they were the only patients in the series not examined by echocardiography. Echocardiographic examination during the acute phase of Kawasaki disease is desirable, as the sensitivity of echocardiography in the detection of coronary artery lesions decreases during the convalescent phase. ${ }^{10} 11$ This is mainly because the aneurysms shrink, and it is impossible to detect stenotic lesions with echocardiography. ${ }^{10} 11$ During the acute illness, coronary artery aneurysms were usually seen during the fourth week, emphasising the need to carry out serial examinations. During the acute phase only three large aneurysms were seen. According to Japanese reports, ${ }^{4}$ only large aneurysms tend to become obstructed, but this study shows that even a moderate sized aneurysm can do so, resulting in myocardial infarction and sudden death.

In those patients who were first examined more than a month after the onset of the acute disease no coronary arterial abnormalities were seen. This does not imply, however, that they had normal coronary arteries. They could have had aneurysms that had regressed, and the coronary arteries may appear normal despite stenotic lesions.

Selective coronary arteriography has not been carried out routinely at this hospital in patients with coronary artery lesions. Echocardiographic examination has been considered to be adequate for the follow up of coronary artery lesions until patients have electrocardiographic evidence, or symptoms, of ischaemia. ${ }^{8}$ Electrocardiographic signs of ischaemia are unreliable, however, and if present imply severe disease. ${ }^{12} 13$ Unfortunately, the sensitivity in detecting myocardial ischaemia on an exercise loaded electrocardiogram is also low, ${ }^{13}$ and ventricular hypokinesis on echocardiography means there has been a myocardial infarction, ${ }^{10} 11$ by which time it is too late for intervention. Furthermore, children who do not have symptoms of ischaemic heart disease may die quite suddenly of infarction as a result of obstructive lesions. ${ }^{11}$

It seems therefore that adequate information about stenotic lesions or myocardial ischaemia may not be obtained without selective coronary arteriography ${ }^{12-14}$ or thallium myocardiography. ${ }^{15}$ There is still debate, however, about whether the reliability of coronary angiography outweighs the benefits that accrue from it.

Some of the patients reported in this retrospective study have reached school age and are participating in physical education without being assessed for the presence of stenotic lesions in their coronary arteries. This is a cause for some concern, and in the light of Japanese experience may emphasise the need for a possible modification in the follow up protocol of affected patients. This is especially important for children who had large aneurysms in the acute phase of their disease; they may need not only echocardiography and exercise loading electrocardiography, but also thallium myocardial imaging or selective coronary arteriography, or both, before starting school. Such patients should not take part in competitive sports, and should have a cardiac evaluation every six months. Children with small aneurysms should be reviewed at least once a year. Inhibiting platelet aggregation by giving aspirin, possibly with dipyridamole, is recommended in all patients with abnormal coronary arteries. The long term management, however, has to be adapted for individual patients and determined by the state of their coronary arteries.

Financial support was kindly provided for AS by The National Cardiovascular Center, Osaka, Japan, and for EJT by John Herring and Friends and the Charlotte Parkinson Research Fund (Child Health Research Appeal Trust).

1 Kawasaki T. A new infantile acute febrile mucocutaneous lymph node syndrome. Fapanese Fournal of Allergy 1967;16: lymph node

2 Naoe S, Masuda H. Kawasaki disease as a risk factor of juvenile arteriosclerosis. Domyakukouka 1981;9:27-31.

3 Kamiya T, Suzuki A, Kijima YEA. Coronary arterial lesion in Kawasaki disease: occurrence and prognosis. Recent Advances in Cardiovascular Disease 1982;III:19-27 (Japanese).

4 Suzuki A, Kamiya T, Ono YEA. Follow up study of coronary artery lesions due to Kawasaki disease by serial coronary arteriography in 200 patients. Heart Vessels 1987;3:159-65. 5 Tizard EJ, Suzuki A, Levin M, Dillon MJ. Clinical aspects of 100 patients with Kawasaki disease. Arch Dis Child (in press).

6 Kamiya T, Kawasaki T, Okuni M, et al. Report of subcommittee on standardization of diagnostic criteria and reporting of coronary artery lesions in Kawasaki disease. Tokyo: Japanese Ministry of Health and Welfare, 1984:1-10.

7 Modi N, Syme J, Keay AJ, et al. Infection and immunology in the mucocutaneous lymph node syndrome: a report of in the mucocutaneous lymph node syndrome:

8 Novelli VM, Galbraith A, Robinson PJ, et al. Cardiovascular abnormalities in Kawasaki disease. Arch Dis Child 1984;50: 405-9.

9 Matsuo H, Matsumoto M, Hamanaka Y. Echocardiographic imaging of coronary aneurysm due to mucocutaneous lymph node syndrome (MCLS). Fapanese Fournal of Medical Ultrasonics 1977;31:139-40 (Japanese).

10 Suzuki A. Standardization of diagnosis of coronary aneurysm due to Kawasaki disease by echocardiographic examination. In: Baba K, Kobayashi, Kato H, eds. Diagnosis of children's disease by echocardiography. Tokyo: Kanehara Ltd, 1986:159-70 (Japanese).

11 Nakaya S, Houjou T, Sugiyama HEA. Problems of estimation of coronary artery lesion due to Kawasaki disease by echocardiography: about false positive and false negative. Proceedings Kinki Kawasaki disease research conference Tokyo: Kinki Kawasaki

12 Suzuki A, Kamiya T, Ono Y, et al. Myocardial ischemia in Kawasaki disease: follow-up study by cardiac catheterisation and coronary angiography. Pediatr Cardiol 1988;9:1-5.

13 Teraguchi M, Yazawa K, Yoshibayashi MEA. Myocardia ischemia on children with severe stenotic coronary artery lesion due to Kawasaki disease. Progress in Medicine 1988;8: 69-75 (Japanese)

14 Suzuki A, Kamiya T, Kuwahara NEA. Coronary artery lesions of Kawasaki disease: cardiac catheterisation findings of 1100 cases. Pediatr Cardiol 1986;7:3-9.

15 Mitomori T, Yasuo $\Theta$, Sugiyama $\mathrm{H}$, et al. Myocardial ischemia due to Kawasaki disease on myocardial imaging thallium ${ }^{201}$ myocardial imaging at rest and at exercise load ing. Cardiography 1984;14:59-73 (Japanese). 